\title{
FACTORES CLAVE PARA EL DESARROLLO Y LA ESTABILIDAD ECONOMICA EN EL PERU
}

\section{KEY FACTORS FOR DEVELOPEMENT AND ECONOMIC STABILITY IN PERU}

\author{
Zelma Wong TorRes ${ }^{1}$ \\ Docente Asociada de la Facultad de Ciencias Contables, UNMSM \\ Luisa Elena Salcedo Guzmán ${ }^{2}$ \\ Docente Asociada de la Facultad de Ciencias Contables, UNMSM
}

(Recepción: Agosto de 2009 / Conformidad: Octubre de 2009)

\section{RESUMEN}

Los resultados de la investigación realizada, pretende ser un modelo referencial y motivador para lograr nuevas formas de enfrentar los retos del desarrollo. Hemos analizado los factores que son clave para el despegue económico y sostenible del país, pues, conociendo el pasado y el presente se puede construir las bases para un futuro mejor. La universidad, juega un papel importante en el desarrollo de la economía de un país, principalmente por las investigaciones e innovaciones que surgen de cada trabajo realizado por los diferentes profesionales en su área de investigación. Por ello, las universidades deben crear los mecanismos necesarios y participar activamente con las empresas y el gobierno en las especialidades de educación, contabilidad, economía, administración, ingeniería electrónica, ingeniería industrial, matemáticas, informática, entre otras muchas disciplinas que se dan en las universidades y lograr el apoyo técnico especializado, que no se encuentra articulado, por falta del enlace entre Universidad, Empresa y Estado. En conclusión, nos falta el acercamiento para promocionar lo que tenemos y valemos.

Palabras clave:

Neoliberalismo, Conocimiento y Educación, Cultura, Competitividad, Identidad Nacional, Potencialidades, Desarrollo Económico Regional

\begin{abstract}
The results of the investigation pretend to be a reference and motivational model to achieve new ways of tackling development challenges. We have analyzed the factors that are key to the sustainable economic revival of the country, because, knowing the past and present it is possible build the foundation for a better future.

The university plays an important role in developing the economy of a country, mainly by research and innovations that arise out of work performed by different professionals in their area of research. That is why the universities should provide the necessary mechanisms and participate actively together with companies and government in the fields of education, accounting, economics, management, electronic engineering, industrial engineering, mathematics, computer science, among many other disciplines that exist in the universities, and to achieve specialized technical support, that is not articulated, for lack of liaison between universities, companies and State. In conclusion, we lack the correct approach to promote what we have and what we worth.
\end{abstract}

\section{Key Words:}

Neoliberalism, Knowledge and Education, Culture, Competitiveness National Identity, Potentialities, Regional Economic Development.

Magisten en Ciencias Contables, UNMSM. Contadora Pública Colegiada Certificada. Docente Investigadora de la Facultad de Ciencias Contables, UNMSM. E-mail: zwongt@hotamail.com

Contadora Pública Colegiada. Docente Investigadora de la Facultad de Ciencias Contables, UNMSM.

E-mail: luisalcedog@yahoo.es 


\section{INTRODUCCION}

En Perú se ha generado estabilidad económica con desigualdad social y exclusión, lo que se hace latente en los conflictos y violencia social, que existen actualmente, no hay fluidez en la movilidad social, sobre todo en el mercado laboral, porque el Estado no ha logrado reformarse y reestructurarse fiscalmente, no ha generado el principio de autoridad necesario para arbitrar conflictos.

En 1990 el péndulo peruano se movió en lo económico, del populismo heterodoxo al neoliberalismo ortodoxo; los cuatro gobiernos que se han sucedido últimamente han mantenido los mismos principios básicos.

$\begin{array}{ll}\text { Alberto Fujimori Fujimori } & 1990-2000 \\ \text { Valentín Paniagua Corazao } & 2000-2001 \\ \text { Alejandro Toledo Manrique } & \text { 2001-2006 } \\ \text { Alan García Pérez } & \text { 2006-hasta la fecha }\end{array}$

Cabe señalar que Fujimori, que carecía de un plan de gobierno, aplicó la receta del Consenso de Washington.

La aplicación del «Consenso de Washington» en un país como Perú, con un ex Presidente sin plan de gobierno, con un sistema político debilitado, con desigualdad distributiva y fragilidad en todo orden de cosas, tuvo la puerta abierta en nuestro país.

El Consenso de Washington, fue creado como un documento de trabajo de Jhon W. Williamson en 1989, economista del Instituto Internacional de Economía, quien lo llama su hijo ilegítimo. Sus padrinos el Fondo Monetario Internacional (FMI), el Banco Mundial y el Departamento del Tesoro de los Estados Unidos.

Es un decálogo que empieza con un adoctrinamiento, es decir, nuevas formas de subordinación y control enmascaradas en una política de reformas.

El Congreso de la República se negaba a apoyar tales medidas, por lo que dicha negativa sirvió de pretexto para que el 05 de abril 1992 el ex Presidente Fujimori se infringió un auto-golpe cerrando el Congreso, interviniendo muchas instituciones, entre ellas al poder judicial, es a partir de aquí, donde se estableció un régimen neoliberal en lo económico y autoritario en lo político.

Que contenía esta receta:

1. Disciplina Presupuestaria.- No más déficit fiscal, tener un presupuesto balanceado, es decir, presupuestos equilibrados; dado que los trastornos macroeconómicos son las consecuencias de los procesos inflacionarios.
2. Reorientación del gasto público-- Desde los subsidios indiscriminados, a actividades ineficientes, hacia la eficiencia en salud, la enseñanza primaria y una mayor infraestructura.

3. Reforma Fiscal.- Encaminada a aplicar la base imponible y mantener tipos marginales moderados. Fuertes impuestos a empleados y empleadores. Imposición de políticas tributarias autodestructivas para que los inversores compraran los recursos claves a precio de remate.

Esta responsabilidad fiscal, válida para todos, excepto los Estados Unidos.

4. Liberación Financiera.- Sobre todo en lo relativo a los tipos de intereses y las ganancias de capital. Control de la inflación como parámetro central de la economía, manejo de la política monetaria, vía tasa de interés, encareciendo y limitando la oferta monetaria sin marco regulatorio adecuado.

5. Tipo de Cambio Competitivo- Los países deben tener un tipo de cambio competitivo para promover las exportaciones, se debe eliminar el proteccionismo arancelario para no hallar trabas al acceso libre de los productos y, aplicar los flujos financieros.

6. Apertura Comercial.- Apertura de los mercados a los inversionistas acompañados de altos tipos de intereses. Dependencia Internacional de Libre Mercado. Dependencia comercial, financiados como créditos en moneda extranjera destruyendo el empleo y aumentando la pobreza

7. Liberación de la inversión Directa Extranjera.- La inversión busca mercados en crecimiento para complacer a los bancos de Estados Unidos y a los inversionistas extranjeros. Inversionistas globales que comprarán las industrias claves.

8. Privatización de Empresas Públicas.- Para justificar estas privatizaciones se divulgó a través de los medios de comunicación que las empresas privadas eran más eficientes que las entidades públicas. Se aplicó esta receta sin políticas de competencia y vigilancia.

9. Desregulación.- Se refiere a la eliminación de barreras de entrada y de salida en los mercados de trabajo y de producción.

10. Derechos de Propiedad Privada.- Garantizando, especialmente en el sector informal.

Estados Unidos no tiene la menor intención de aplicar este modelo en su propia economía.

Estas medidas se ajustan perfectamente a las prescripciones de la contrarrevolución neoclásica radical o neoliberal de los ochenta y el enfoque neoclásico moderado o liberal de los años noventa. 
El Consenso de Washington era para lograr e incrementar y consolidar la fortaleza e influencia de Estados Unidos en las últimas décadas.

Teniendo presente que la historia de la humanidad se resume en tres grandes eras:

- La era de la Agricultura

La era Industrial

La era del conocimiento

En el tiempo actual, con las grandes transformaciones y avances de las «sociedades del conocimiento» los cambios son tan vertiginosos, que a muchas sociedades cuesta adaptarse a estos cambios. Como producto de estas transformaciones, ya podríamos estar vislumbrando una nueva civilización en el mundo.

La fuerza laboral que hoy en día requieren las empresas, va dirigida no a tener mayor número de trabajadores, sino, a la inteligencia y capacidad de aprender e innovar, pues el trabajo que carece de contenido mental en estos tiempos, ya no tiene cabida, definitivamente el valor real de los productos, radica en el conocimiento que se le incorpore.

El Perú tiene una coyuntura internacional muy favorable y cambios en la estructura productiva, queda evaluar qué factores no permiten entrar al círculo virtuoso del crecimiento y estabilidad económica con mayor equidad y gobernabilidad y qué futuro tiene el Perú de alcanzar mejores condiciones de desarrollo humano.

Por todo esto, hemos llegado a la conclusión que los factores que promueven el desarrollo son:

1. Conocimiento y educación.

2. Cultura.

3. Competitividad

4. Identidad Nacional

5. Potencialidades

6. Desrrollo Económico Regional

\section{CONOCIMIENTO Y EDUCACION}

El conocimiento ha sido factor clave para el desarrollo y progreso de la humanidad, toda transformación requiere de conocimiento, sin el conocimiento no es posible producir absolutamente nada, por ello la educación es un factor elemental en el desarrollo del conocimiento, para desarrollar la capacidad de innovación y creación entre las personas de una sociedad.

El incremento de la inversión en ciencia y tecnología son fundamentales para la transformación y crecimiento del país. Si se mejora e implementa políticas educativas adecuadas a nuestra realidad, se conseguirá desarrollar los recursos humanos, que son la base del crecimiento de toda sociedad.
UNESCO, CEPAL y el BANCO MUNDIAL, han diagnosticado que las universidades en América Latina, vienen atravesando una crisis severa, relacionada con factores socio-económicos inherentes a cada país, señalando específicamente que la falta de calidad en las universidades, se debe a tres factores claramente diferenciados:

- Incremento de instituciones que no reunen los requisitos y exigencias mínimas de trabajo académico.

- Falta de preparación del personal dedicado a las labores académicas, en especial a la investigación y docencia.

- Metodología académica anacrónica que vincula el aprendizaje con la transmisión repetitiva al alumno.

La universidad peruana se encuentra desfasada con el fenómeno de la globalización, que exige la acreditación internacional, la educación impartida en las aulas no está teniendo en consideración las exigencias y oportunidades laborales y mucho menos con posibilidades de contribuir al desarrollo nacional, que se alcanza por medio de las investigaciones.

El mundo económico reclama cada vez más calificaciones y competencias, el mundo científico reclama créditos para la investigación y para la enseñanza superior de alto nivel, productora de jóvenes investigadores; el mundo de la cultura y de la enseñanza requiere medios para el desarrollo de la formación en general, además, los padres de los alumnos reclaman un aumento constante en la calidad de la educación.

Frente a esto hay que estandarizar la educación y definir un solo proyecto educativo internacional, pues existe mucha diversidad de situaciones, concepciones y estructuras de la educación, tanto, regional como a nivel nacional.

Para que la educación tenga carácter internacional se debe estandarizar el currículo homogenizando el mismo.

El objetivo de toda política educativa es el aprendizaje de una sociedad determinada, objetivo que se extiende hacia la educación con participación, solidaridad, e integración. Siendo así, la política educativa es un acto del cual debe encargarse el gobierno, plasmando consensos sobre un futuro ideal.

La lógica que articula las contrarreformas educativas la muestra como, coherentes y conducentes en busca de un punto común.

Es sabido que países desarrollados, no cuentan necesariamente con potencial agropecuario, su gran potencial se basa en el desarrollo de tecnologías, por ello, producir nuevos conocimiento es base 
importante para el desarrollo del país. Para producir nuevos conocimientos y nuevas técnicas productivas se debe invertir en investigación científica y tecnológica teniendo en consideración las MYPES.

De comienzo a fin, el actor principal del desarrollo, son los seres humanos; si el Estado no apunta paralelamente al bienestar material y espiritual de las personas. Si no pone freno a la recesión productiva, terrorismo, violencia social, autoritarismo y corrupción en general, la autoestima de los peruanos se deteriora.

Los múltiples factores geográficos, legales, sociales, étnicos y culturales, han hecho que en el Perú la igualdad ciudadana todavía sea una meta a alcanzar y no una realidad, lo cual exige mayores niveles de organización para participar en la política y en la sociedad.

Entre los países vecinos, el que invierte más en investigación y tecnología es Brasil que al 2007, invirtió en investigación 101.44 dólares por habitante.

Según información de la Red de Indicadores de Ciencia y Tecnología (RICYT) el Peru invierte en investigación y desarrollo (I+D) menos de cuatro dólares anuales por habitante el 2004, situación que se viene aplicando hasta el 2009.

Con esta situación podemos comprender, porque nuestro país compra, y a precios muy altos, el conocimiento. Somos dependientes de las tecnologías extranjeras, lo que nos hace más y más pobres. Invertir en ciencia no es políticamente rentable, ya que es una inversión a mediano y largo plazo, si nos fijamos en países como Corea del Sur o Irlanda, que apostaron por la ciencia y tecnología y cuyos frutos los están cosechando las generaciones presentes y futuras.

Resultado, nos encontramos con un país, carente de política científica y por ello la «fuga de talentos».

Frente a todo esto, tenemos otros indicadores que nos van diciendo como andamos, entre ellos tenemos el Índice Gini, es uno de los principales indicadores de la distribución de los ingresos.

Cuanto más cerca sea este valor a 1, significa que peor es la distribución del ingreso, en tanto cuanto más cercano sea este valor a 0 , indicaría que la distribución de los ingresos es más equitativa. Así tenemos que:

\begin{tabular}{|c|c|}
\hline Año & I. Gini \\
\hline 1997 & 0.473 \\
\hline 1999 & 0.498 \\
\hline 2001 & 0.477 \\
\hline 2003 & 0.456 \\
\hline 2004 & 0.492 \\
\hline 2005 & 0.508 \\
\hline 2006 & 0.499 \\
\hline 2007 & 0.507 \\
\hline 2008 & 0.479 \\
\hline
\end{tabular}

Comparando el periodo de Toledo tenemos que el índice Gini ha pasado de 0.477 en el 2001 al 0.499 en el 2006. El coeficiente Gini sólo recoge sueldos y salarios y deja de lado los ingresos por capital (ganancias interés y rentas de las personas).

Observamos que en periodo 1997 al 2008, el menor índice se registra en el año 2003, durante el gobierno de Toledo.

Los países con la mejor distribución de ingresos en el mundo son Holanda, Dinamarca, Eslovaquia, Países Nórdicos, Australia y Japón.

Estos países tienen indicadores de Gini por debajo de 0.25 , es decir, que nuestro país tiene todavía mucho que aprender y mejorar.

La desigualdad se ha mantenido, incluso ha aumentado en los niveles altos de manera muy visible y al mismo tiempo en los niveles bajos aumento de la mendicidad callejera, la emigración y la delincuencia entre otros

Para disminuir la desigualdad es necesaria una capacidad distributiva más equitativa.

Toda organización, para salir adelante debe contar con una excelente estructura organizativa, pero aún más importante es contar, entre sus miembros con buenos líderes.

El liderazgo es la capacidad de dirigir a otros con éxito, el líder tiene características propias. El líder debe tener poder de manejo, alta conductividad y poder de absorción; mediante esto se logra mejor aprovechamiento de las capacidades de los trabajadores por lo que toda organización está en busca de líderes. El líder tiene personalidad y seguridad de lo que expresa y hace, no es una persona que se expresa a medias tintas e incoherentemente.

Otra área a la que se debe dar atención son los descubrimientos científicos, la invención y la 
patente registrada estos son los que generan dinero al científico, y al país en donde se desarrolla el invento; son éstas las que dan cuenta de la marcha de la ciencia en un país, analicemos pues los indicadores:

\begin{tabular}{|l|c|c|c|c|c|c|c|}
\hline \multicolumn{1}{|c|}{ Países } & $\mathbf{2 0 0 2}$ & $\mathbf{2 0 0 3}$ & $\mathbf{2 0 0 4}$ & $\mathbf{2 0 0 5}$ & $\mathbf{2 0 0 6}$ & $\mathbf{2 0 0 7}$ & $\mathbf{2 0 0 8}$ \\
\hline Estado Unidos & 265 & 237 & 186 & 127 & 103 & 110 & 125 \\
Brasil & 12 & 7 & 6 & 3 & 5 & 3 & 5 \\
Chile & 1 & 4 & 2 & 1 & 2 & 3 & 6 \\
Argentina & 10 & 3 & 5 & 2 & 2 & 4 & 0 \\
Colombia & 0 & 0 & 1 & 0 & 1 & 3 & 0 \\
\hline Perú & $\mathbf{2 2}$ & $\mathbf{1 6}$ & $\mathbf{1 3}$ & $\mathbf{5}$ & $\mathbf{5}$ & $\mathbf{1 5}$ & $\mathbf{5}$ \\
\hline
\end{tabular}

Como podemos apreciar es muy pobre nuestro registro de patentes.

Los países asiáticos son los que registran anualmente cientos de patentes de invención en la organización mundial de propiedad intelectual.

\section{CULTURA}

Otro factor que debemos tener en cuenta es la Cultura:

La cultura es la memoria de la sociedad, producto de años de creación e innovación, en la que la sociedad basa sus objetivos futuros. Por ello, la cultura es un factor fundamental del desarrollo de un país.

En la globalización la identidad cultural es justa y necesaria para marcar las diferencias, uno de sus rasgos es ser motor de crecimiento, acumulación de saberes y capacidad creativa colectiva.

El escenario global confronta a la sociedad con sus enormes desafíos, debemos respetar nuestra propia identidad, sino se logra esto nos podemos estancar y desarticular.

La cultura en nuestra patria, debe ser revalorizada, y tenerla en cuenta en los planes de desarrollo.

La identidad cultural contribuye a la liberación de los pueblos; el Instituto Nacional de Cultura (INC) juega un rol muy importante y trascendente en la afirmación de la identidad nacional, ejecutando políticas y estrategias en materia de desarrollo cultural, que será el motor que dinamice y fortalezca el crecimiento económico, político y social de Perú.

El gran problema del país no es económico, sino más bien, cultural, notemos que el 10\% de los científicos del mundo están en países desarrollados, de éstos el 90\% se encuentran distribuídos entre Taiwán, Corea, Singapur, Malasia, China e India. Después del hallazgo científico que realizara Daniel Alcides Carrión hace 124 años, en el Perú no se han registrado patentes de gran magnitud.

A la fecha, los proyectos de investigación más destacados son los que se remiten al estudio de propiedades de plantas y sustancias químicas.
La inequidad es una de las características del gasto público en educación.

El Estado invierte mucho menos en regiones en donde el nivel de pobreza económica es muy alto. En otras palabras, el presupuesto del sector sigue siendo centralista y con una distribución carente de criterio.

Es evidente que la insuficiencia de recursos presupuestales tiene un impacto negativo sobre la calidad de la educación, pues a menor presupuesto, menor capacidad de adquirir material didáctico. Notamos que no hay coherencia entre meta de estudiantes atendidos y tamaño presupuestal.

La capacidad de generar conocimiento por medio de la investigación e innovación son medidos en indicadores que muestran que la inversión en este rubro es pequeña.

Después que el Congreso aprobara el presupuesto público 2009 ascendente a 72,000 millones de nuevos soles, nos dimos con la sorpresa que el Perú invierte el $0.001 \%$ de su PBI en investigación.

\section{COMPETITIVIDAD}

Nuestro país no está lejos de ser un país competitivo, y abierto al mercado global, todo esto lo podemos conseguir si se reactiva el Instituto Nacional de Planificación (INP)con proyectos de corto, mediano y largo plazo para que al aplicar de forma ordenada dichos proyectos una vez investigados para su convalidación, lograr resolver en forma escalonada los problemas que afronta nuestro país. Nos hallamos en esta situación, por carecer de una institución planificadora y por falta de proyectos de desarrollo a corto, mediano y largo plazo, es allí en donde radica nuestro sub-desarrollo.

Debemos diseñar un proyecto Nacional en todas las escalas micro y macro.

Un proyecto nacional es donde intervienen todos los sistemas que componen la sociedad. 
El Proyecto Nacional debe ser diseñado por un equipo de especialistas en ciencias y técnicas sociales y biosociales, antropólogos, demógrafos, sociólogos, culturológos, historiadores epidemiólogos, expertos en administración de empresas, contadores, juristas y pedagogos; el proyecto es para el pueblo, el gobierno y la combinación de ambos.

Perú sigue estancado debido a que las instituciones públicas no son sólidas ni creíbles. No se ha dado la tan ansiada reforma del Estado.

La economía mundial afronta grandes cambios, enmarcados en la globalización, avances científicos, tecnológicos, desarrollo de la comunicación, y la constante demanda de productos de alta calidad que incentiva a la competencia de mercados.

Debemos enfocarnos a implementar políticas diseñadas para el desarrollo socio-económico de la nación, con la finalidad de poder ser competitivos internacionalmente.

Aunque el Estado, apoya el desarrollo de las empresas nacionales, mediante leyes y programas de crecimiento a las MYPES, es mucho lo que falta aún por hacer, se debe capacitar al microempresario para producir bienes y servicios de manera eficiente, en los que sobresalga la calidad, para esto se requiere elevados niveles de capacitación.

En el ranking mundial de competitividad, Perú se ubica con posiciones muy expectantes, esto, debido al buen desempeño económico, sin embargo, referente a la competitividad aún quedan rubros en los que se debe mejorar, entre ellos la eficiencia del gobierno, eficiencia de los negocios e infraestructura.

El gobierno tiene como tarea la implementación de políticas que mejoren la competitividad en infraestructura vial y de telecomunicaciones, mejorar los muelles y puertos, no sólo los cercanos a la capital sino los dirigidos a la descentralización.

Ampliar las redes eléctricas de los distintos centros poblados, realizar estudios para el suministro de energía eléctrica a las diferentes regiones, entre otros.

Se debe definir el modelo educativo con las competencias, y en particular las llamadas «aprender a hacer» las mismas que se combinan con los conocimientos teóricos y prácticos para poder acceder a las competencias que se solicitan.

La burguesía está imponiendo la idea de convertir la educación en una mercancía y con los TLC busca que sea de fácil circulación.

El imperialismo busca por todos los medios mantener la función del sistema educativo como aparato ideológico del estado, porque la escuela es el lugar por excelencia de transmisión e imposición de ideas y culturas dominantes sin desconocer el papel de uno y otro.
No es tiempo de lamentarnos de las competencias técnicas y profesionales acumulados a lo largo de la vida, pero sin utilidad para el mundo real, nos quedamos estancados a pesar de los conocimientos, faltó desarrollar competencias personales, laborales, empresariales y manejo ejecutivo, no se ha manejado adecuadamente, pese que allí estuvo y esta esperándonos. Hasta la actualidad solamente se ha destruido, para mantener nuestros puestos de trabajo porque si nos despiden no sabemos hacer nada más y eso es lo que se está transmitiendo a los jóvenes estudiantes, futuro de la nación, a ser uno más del montón, es necesario para despertar y no hacernos daño unos contra otros, siendo hermanos trabajemos juntos, evitemos que otros países se lleven lo que es nuestro; que no aprovechamos en forma efectiva por los simples líos domésticos sin trascendencia profesional.

Podemos establecer las siguientes competencias:

Competencia Personal.- No laboral, no empresarial. Se refiere a la capacitación para ser especialista real y objetivamente, no sólo de título o de palabra. Esto se consigue, mediante la acreditación con los grados y títulos, no por compañerismo ni por política, cada persona debe conocer su valor, conocer a la perfección lo que realmente sabe y vale, su grado de competencia, no prestar oídos a los negativos que echan por tierra y anulan el crecimiento, porque la incompetencia se une para destruir y en política se crean falsos éxitos, sin bases, ni conocimientos.

Competencia Social.- Todo lo adquirimos con el paso de los años por la experiencia que se adquiere y el roce social. La educación es la vía para cambiar, pero qué tipo de educación, aquella que tiene aplicación rápida y práctica.

Debemos propender a ello sin descuidar la informática, los idiomas como el inglés, chino mandarín, hindú y otros, pues para mostrar competencia debemos comunicarnos en el idioma del país con el que estoy comercializando, por lo tanto, no se trata de tener personal calificado, sino además, que domine el idioma del país con el que se transará negocios.

Debemos tener un Estado solvente a nivel profesional, eficaz y que cuente con los mejores y más calificados egresados de universidades a fin de fijar claramente los objetivos nacionales.

Competencia Laboral.- Este tipo de competencias se refiere a los valores que se deben practicar tales como: la puntualidad, la higiene, la responsabilidad, la perseverancia, la honradez, la moral, la ética, la honestidad, entre otras No se tiene en la actualidad un programa o institución que brinde cultura laboral. 
Competencia Empresarial o Manejo Ejecutivo- Hay en el país instituciones que brindan cursos relacionados a la gestión empresarial; sin embargo, las personas que los imparten no cuentan con la experiencia empresarial práctica necesaria, basándose en la enseñanza por medio de libros de otras autorías, de otras realidades, sin aplicación a nuestra propia realidad, en la mayoría, casos extranjeros. Nos preguntamos ¿qué es lo que enseñan? más aún, y ¿qué tipo de especialistas o ejecutivos se consigue formar finalmente?. Pues, simplemente teóricos de otras realidades no aplicables a nuestro entorno, sin práctica alguna, y sin capacidad competitiva.

Estas competencias antiguamente no se estudiaban, se heredaban de padres a hijos, pero el mundo está cambiando, cambiemos nosotros, transmitiendo a los estudiantes de hoy (futuro del país) conocimientos reales.

Que cada peruano logre ser un empresario no un dependiente, de lo contrario seguirá no habiendo campo para los jóvenes, pues al no contar éstos con experiencia, las empresas actualmente demoran las jubilaciones de sus trabajadores experimentados, porque los nuevos trabajadores carecen del conocimiento suficiente y capacidad emprendedora.

Frente a todo lo mencionado nos preguntamos Que nos trae la Globalización?

Algunas características de la globalización son:

- Transnacionalización de los procesos nacionales.-

Los converge en uno solo y universal en el plano económico, social, jurídico y tecnológico, surgiendo un nuevo espacio trasnacional unificado que incluye también la parte delictiva, ahora multiplicado y acrecentado con nuevas dimensiones del terrorismo, narcotráfico, crimen organizado o Lavado de dinero amenazas propias del proceso de transnacionalización que afecta a los estados y al sistema en general.

- Fortalecimiento de fuerzas locales.- que se resisten al proceso de cambio basados en el mantenimiento de la identidad de intereses tradicionales.

- Surgimiento de la economía de la información y transformación de los procesos conflictivosimplican un manejo de coerción racionalizada de estado.

- Influencia de actores no estatales.- Representan intereses parciales o de grupo empresariales.

- Nueva fase de realidad internacional.- Crear organismos sistémicos.

\section{IDENTIDAD}

Entre las ventajas competitivas que posee el Perú tenemos que está geopolíticamente muy bien ubicado, lo que constituye una ventaja sobre otros países. El Perú es considerado por el mundo como una zona de amortiguamiento, es decir, zona limpia y protegida.

Perú es el tercer país más grande de América del sur, después de Brasil y Argentina, siendo su capital Lima, principal centro de poder político, comercial y financiero.

Para desarrollar la identidad nacional en Perú, se requiere cooperación social, afianzar el sentido de pertenencia, tener un claro concepto de nación y nacionalidad, asociados a elementos como la raza, la etnia, religión, geografía, entre otros.

Es necesario, un proceso de recuperación de lo nuestro, de las raíces culturales, revalorizar la tradición. Esta recuperación, se vincula con la revalorización de la investigación, tecnologías, organización y distribución económica. La influencia con los países limítrofes es importante por su posición geográfica; estamos en el centro del mundo y debemos de tomar riendas de nuestro país por ser el centro de tránsito para el comercio mundial, convirtiéndose en una ventaja competitiva no antes vista. En el Perú brota cualquier tipo de semillas con solo dejarla caer. Poseemos 84 (75\%) zonas de vida de las 112 que existen en el planeta, todos los climas y zonas geográficas, siendo esta una ventaja estratégica, según el futurólogo norteamericano Alvin Toffler, quien manifiesta que los países ricos en biodiversidad serán muy importantes.

La Identidad Nacional permite reconocer al Perú como un país plural y heterogéneo que acepta su diversidad cultural y en el cual el respeto a las diferencias es el fundamento del sentido de pertenencia.

El sentimiento de peruanidad, surgido dificultosamente en el contexto de las luchas anticoloniales, se ha ido extendiendo y asentado durante los dos últimos siglos en medio de agudos conflictos y transformaciones, tales como, las sucesivas guerras externas, las guerras civiles, el afianzamiento del desarrollo capitalista, las movilizaciones indígenas y campesinas, las grandes migraciones que urbanizaron el rostro del país, las reformas agrarias y el proceso de violencia interna de las dos últmas décadas del siglo XX.

\section{POTENCIALIDADES}

Se entiende como potencialidades a todos aquellos recursos que existen en una determinada zona, pero que no están siendo utilizados. 
Hay dos tipos de potencialidades tangibles e intangibles.

Dentro de estas potencialidades podemos establecer

- Potencial Agrario

- Potencial Agropecuario

- Potencial Minero

- Potencial Hidroenergético

\section{Potencial Agrario:}

Una característica importante es que en las regiones que observamos, existen mayores potencialidades de suelos, pastos y forestales, lo que indica un uso más racional y una relativa mejora tecnológica.

Puno tiene posibilidades agropecuarias que puede aún desarrollar, especialmente, por disponer de capital humano por utilizar.

El caso particular de Junín, es la dominación de la producción por la refinería de la Oroya, lo que tiende a aparentar una estructura esencialmente industrial, aun cuando su actividad agropecuaria, segunda en orden de importancia, es un complemento de orden mayor, para el empleo. Se trata, además, de un sector agropecuario más tecnificado y vinculado comercialmente con Lima.

Dentro de las Potencialidades Agropecuarias observamos que en las últimas décadas la actividad agropecuaria en el Perú era principalmente para el consumo interno y aún así teníamos déficit de algunos productos, tales como, el trigo y el maíz, esta situación se ha revertido gracias a un franco crecimiento estos últimos años en la actividad de la agro exportación.

Algunos datos estadísticos y oportunidades indican que esta actividad económica será uno de los pilares de nuestro desarrollo.

No obstante, si bien existen muchos productos originarios del Perú que se pueden cultivar eficientemente en la sierra para los que sí existen mercados foráneos, el problema es que no son mercados en crecimiento. Así, los expertos sugieren un desarrollo inverso: buscar qué productos con mercados en crecimiento, pueden cultivarse eficientemente en la sierra peruana.

Si bien la Sierra peruana no tiene las favorables condiciones comparadas con la costa, ni en clima, ni en las extensiones, ni el manejo de cultivos; pero a pesar de esas dificultades, su potencial agro exportador no es nada desdeñable, y ya hay iniciativas en esa dirección. Actualmente existen intentos de exportación desde la sierra -la mayoría pequeños- de productos agrarios no tradicionales. La alcachofa y la quinua se exportan en cantidades considerables, y el anís (Apurímac), la fibra y carne de camélidos sudamericanos, el maíz blanco, la cochinilla, maca (en Junín y Cerro de Pasco), entre otros, se exportan en cantidades pequeñas pero con potencialidades futuras.

\section{Potencial Minero:}

La minería es uno de los sectores más importantes de la economía peruana y representa normalmente más del $50 \%$ de las exportaciones peruanas con cifras alrededor de los 4 mil millones de dólares al año. Por su propia naturaleza, la gran minería constituye un sector que genera grandes movimientos de capital más no de mano de obra, sin embargo, ocupó sólo al 0.7\% de la Población económicamente activa (PEA).

Los principales minerales que exporta el Perú son: cobre, oro, hierro, plata, zinc y plomo entre otros. Actualmente todos ellos son fuertemente demandados como insumos para procesos industriales de alto nivel tecnológico.

\section{Potencial Hidroenergético:}

El Perú cuenta con un potencial teórico de 56.000 mega watios de energía. Si se pudiese expresar de manera sencilla tal potencial hidroenergético, podríamos afirmar que la capacidad energética del Perú es tal que podría encender las luces de todos los países de Sudamérica a excepción de Brasil.

De esos 56.000 mega watios en la actualidad sólo se encuentran plenamente explotados 2.000 mega watios. Algo curioso que cabe citar es que, a pesar de contar con tal potencial no todos los pueblos del país se encuentran electrificados y esto obedece a una razón: la energía es un bien que se vende y como tal las empresas requieren que los usuarios tengan una capacidad de pago que muchas comunidades no poseen.

Más contradictorio aún, es el hecho que la Región de Huancavelica, siendo la que más energía eléctrica provee al país, es la que cuenta con la menor proporción de personas con conexión eléctrica.

\section{DESARROLLO ECONOMICO REGIONAL}

El Perú es una Nación rica en potencialidades. Sin embargo, se requiere mucho esfuerzo para superar los niveles de pobreza, que afecta a parte significativa de los peruanos. El reto ahora consiste en superar los niveles de desarrollo humano e identificar formas para aprovechar las potencialidades que ofrece cada región.

Éstas potencialidades no son referidas sólo a los recursos naturales, sino, además que comprende los activos o capitales que posee el país, nos referimos al nivel educativo, la herencia histórica, infraestructura educativa, economía, finanzas y desde luego la biodiversidad. 
Tenemos un potencial turístico muy grande debido entre otros, a los nuevos descubrimientos arqueológicos, tales como, la ciudad de Caral, el Señor de Sipán, Choquequirao, El Brujo y otros, son presentados al mundo, analizados a partir de patrones y esquemas interpretativos, que no corresponden a la realidad cultural del mundo andino.

Cusco tiene capacidad hotelera en uso potencial. Una dotación importante de capital humano por utilizar, obviamente en relación a su enorme potencial turístico.

Ancash y San Martín, hay usos y potencialidades comerciales y turísticas. En estas regiones la industria no ha tenido suficiente desarrollo; sin embargo, en turismo, se está desplegando por servicios privados. Es a la vez preocupante la insuficiencia de capital humano. Intensificar la provisión de recursos humanos es una necesidad del propio desarrollo turístico regional, pero, sobre todo, del paso hacia mayores dosis de valor agregado en la producción. A todo esto debemos agregar el sabor de los alimentos peruanos, oferta turística incomparable.

Este año, el 30\% de los turistas que visitó el Perú para ir a Cusco, decidió quedarse en Lima un par de días adicionales sólo por todo lo que había leído y escuchado acerca de su gastronomía. Esto se debe a que los periodistas más importantes de todo el mundo son enviados a cubrir esta revolución culinaria, publican artículos y emiten programas de TV por todo el mundo deslumbrados por lo que ellos consideran la inminente invasión peruana de sabores.

Con relación a las plantas medicinales este es incipiente, sin embargo, se debe propiciar mayor investigación en esta área del saber, aquí se debe concretar que hay que intercambiar conocimientos con los lugareños, pues ellos saben perfectamente para que sirven las variedades de plantas medicinales que poseemos.

El Índice de Desarrollo Humano (IDH), es una medida elaborada por el Programa de Naciones Unidas para el Desarrollo (PNUD). El Indicador está basado en tres parámetros:

Esperanza de Vida al nacer, Educación y Nivel de Vida digno.

Éste índice busca explicar y discutir alternativas sobre las desigualdades en el desarrollo humano, convirtiéndose en un barómetro de las políticas públicas y de la dirección adoptada por las inversiones, la atención que brindan el gobierno y los inversionistas privados; así como la economía nacional y el desaprovechamiento de los recursos locales.

Planteado así, observamos que las regiones con mayor índice de desarrollo humano (IDH), en el Perú son:

\author{
- Lima, Callao, Ica, Arequipa, Moquegua y
} Tacna.

Claramente el Índice de Desarrollo Humano (IDH) de Lima y Callao es superior a cualquier otra región, este fenómeno está asociado con el fenómeno migratorio interno.

En tanto que las regiones con menor índice de Desarrollo humano (IDH) son:

- Amazonas, Puno, Cajamarca, Curco, Huánuco, Ayacucho, Apurimac y Huancavelica.

Las desigualdades y la falta de relación entre el desarrollo humano de áreas geográficas contiguas en el país, muestran la ausencia de integración económica. Los espacios regionales, reúnen ciudades, pueblos y caseríos desintegrados o insuficientemente integrados, como para que se les pueda considerar como partes de una economía espacialmente unificada.

Las distintas regiones fuera de Lima son economías débiles. En resumen, el primer paso para una mejora en el desarrollo del país es la descentralización, tendrá que enfrentarse escalas económicas suficientes como para generar desarrollo humano.

Las crecientes diferencias en el desarrollo de los pueblos del Perú, tienden hacia el doble efecto negativo de un lento ascenso general, paralelo al crecimiento de las desigualdades internas. Lo siguiente a afrontar es el planteamiento de una alternativa, para lo cual, el conocimiento de las zonas oscuras del Índice de Desarrollo Humano (IDH). Zonas donde el progreso es realmente lento y existen escasas oportunidades, para emprender un marco adecuado de políticas en las cuales desenvolver los recursos de manera que se asegure el desarrollo productivo, autónomo, permanente, sustentable y equitativo.

\section{CONCLUSIONES}

- Los principales factores para promover el desarrollo y crecimiento económico del país son: el conocimiento y la educación, la cultura, la competitividad, la identidad nacional, y la explotación adecuada de las potencialidades regionales.

- Para lograr que los factores que promueven el desarrollo se conviertan en realidad de manera multiplicativa, es necesaria la voluntad política, y que la eficiencia, la equidad y la libertad resulten ser los principales motores del desarrollo.

- Perú es un país, polimetálico, posee el mar más rico del mundo, una megadiversidad envidiable, capacidad de servicio y somos netamente creativos, estas cualidades incrementan la posibilidad de ingresar al mercado mundial, 
haciendo valer lo que la naturaleza nos ha prodigado.

- Es igualmente necesario, el surgir de un nuevo tipo de peruano, eliminar las taras que se han convertido en parte de la cultura actual, la impuntualidad, la criollada, el amiguismo, y otros que nos restan seriedad como personas y como investigadores, taras que debemos reemplazar por valores de honestidad, solidaridad, responsabilidad y libertad, para la creación productiva, artística, cultural y espiritual.

- En el ámbito de investigación y desarrollo de tecnología se requiere diseñar un plan de desarrollo científico y tecnológico conjuntamente con la reactivación del Instituto Nacional de Planificación (INP), así como, asignar presupuestos mayores para este ámbito. El promover la política de cooperación internacional incrementa el asentamiento de nuestra cultura.

- El CONCYTEC, requiere retomar su función como organismo rector del Sistema Nacional de Ciencia y Tecnología, y evitar así, el inadecuado uso de los recursos financieros en investigaciones que muchas veces se duplican, debido a que cada Instituto de Investigación diseña sus proyectos independientemente, con criterio individualista.

- El presupuesto que invierte el Estado en educación, debe descentralizarse, pues actualmente invierte menos presupuesto en regiones más pobres, con una total falta de criterio. Esto tiene un impacto negativo en la calidad de la educación.

- Para incrementar la calidad en la educación es imprescindible una adecuada inversión pública, voluntad política y coordinación institucional, a fin de optimizar el uso de los recursos asignados. La distribución de los recursos debe ser calculado en base a realidades, diferenciando costos de diversos programas y actividades; se debe asumir que la inversión en educación tiene rango estratégico en el desarrollo y crecimiento de la economía de nuestro país.

- El sistema educativo, debe enmarcase en la revolución de la comunicación e información, y la globalización económica. Establezcamos cambios e innovemos en investigación, docencia y acreditación.

- Respecto a las potencialidades, podemos centrarnos en el desarrollo del ecoturismo. La agricultura peruana, es el futuro, se debe incrementar la investigación y tecnología de los productos bandera, así como, la búsqueda de nuevos mercados. Una agricultura menos industrializada es más viable a la realidad peruana. Tenemos que capitalizar las riquezas que poseemos para los negocios de nuevos productos químicos, farmacéuticos y alimenticios, para la humanidad; teniendo presente que los futuros tratados de comercio deben hacerse teniendo en cuenta el reconocimiento de nuestros productos y las regalías por los derivados que se originen de ellos.

- Entre las ventajas competitivas de nuestro país, se encuentra nuestra excelente ubicación geopolítica, lo que nos da influencia con los países limítrofes, y constituyéndonos en el centro de tránsito para el comercio mundial. Perú, también posee ventaja estratégica en el rubro de ecología, e incluso, la gran cantidad de ciudadanos Chinos que son emigrantes en nuestro país, nos da ventaja estratégica con China.

- Las diferencias en el desarrollo de los pueblos tienden a un lento ascenso general, paralelo al crecimiento de las desigualdades internas. Es necesaria la identificación de zonas o regiones donde el progreso es lento y existe pocas oportunidades de mejorar. Emprender un marco adecuado de políticas que aseguren el desarrollo productivo, autónomo y permanente sustentable y equitativo.

- Para poder aprovechar los recursos existentes, y cuya explotación permita el desarrollo de la región y por ende de sus pobladores, es necesario vencer los obstáculos de la geografía nacional.

- Mejorar e implementar los modelos educativos con políticas adecuadas a nuestra realidad, incluso regional, lo que permitirá desarrollar recursos humanos que son la base de todo crecimiento económico.

- Respecto a la cultura, urge que el Instituto Nacional de Cultura (INC) se encargue de la difusión y afirmación de la identidad nacional ejecutando políticas y estrategias en materia de desarrollo cultural, motor que dinamizará el crecimiento económico.

\section{REFERENCIAS BIBLIOGRÁFICAS}

1. Ames, Rolando. Movimiento popular y política nacional, Nuevos Horizontes», Tarea, Número 12, 1985, Lima.

2. Anderson, Benedict. Imagined Comunities, Verso Edition and NLR, 1983. London.

3. Ardao, Arturo. Panamericanismo y latinoamericanismo. Fuentes de la Cultura Latinoamericana, Fondo de Cultura Económica, México, 1993.

4. Balbi, Carmen Rosa. 1989. Identidad clasista en el sindicalismo. Su impacto en las fábricas. DESCO. Lima. 
5. Blondet, Cecilia; Degregori, Carlos Iván y Lynch, Nicolás. Conquistadores de un nuevo mundo. De invasores a ciudadanos en San Martin de Porres. IEP. 1986, Lima.

6. Bodemar, Klaus. La Globalización un concepto problemático en nueva sociedad, No. 156, JulioAgosto, 1998, México.

7. Centro de Altos Estudios Nacionales, CAEN, Planteamientos doctrinarios del desarrollo y defensa nacional - Tomo I, 2001

8. Camarena, M. Perú, nuevo Paraiso Laboral. Lima Perú: Imprenta Gráfica David.

9. Cancino Troncoso, Hugo. Nation og national identitet i det post-koloniale samfund i Latinamerika, ca. 1924-1980, en Den Jyske Historiker, No 81, (Agosto, 1998), Universidad de Aarhus, Dinamarca.

10. Cancino Troncoso. ¿Existe una identidad cultural latinoamericana? Las raíces históricas del debate, en Pablo Cristoffanini (Ed.): Identidad y otredad en el mundo de habla hispana, Universidad Nacional Autónoma de México, 1999.

11. Constitución Política de Perú 1979 y 1993.

12. Franco, Carlos. Imágenes de la sociedad peruana: la otra modernidad. Centro de Estudios para el Desarrollo y la Participación (CEDEP). 1991, Lima.

13. Flores, Galindo Alberto. Buscando un inca: identidad y utopía en los Andes. Casa de las Américas. 1986, La Habana.

14. Flores y Luengo. Globalización, comercio y Modernización Productiva.

15. Una mirada desde la Unión Europea ampliacada. Ed. Akal. 2005. Madrid.

16. Fuller Norma, El papel de las clases medias en la producción de la identidad nacional, Pontificia Universidad Católica del Perú.

17. Gonzales de Olarte, E. (2006). Economía Peruana.

18. Gadamer, Hans Georg (1996), Truth and Method, Sheed \& Ward, London.

19. Halperin Donghi. Historia Contemporánea de América Latina, Alianza Editorial, 1972, Madrid.

20. Higgins, Andrew. Enemies close in on China's whizkid. Guardian. 1996.

21. Hobsbawn, E. J., (1994), Nation and Nationalism since 1789, Cambridge University Press.

22. Kynge,James. China Sacude al Mundo. Lid Editorial Empresarial S.L. 2007. Madrid, España.

23. Kynge, James. Hope holds out for triumph over adversiry. Financial times. 1998.

24. Kurtenbach, Eliane. China's tiny automakers set their sights on the whole world. 2005.

25. López, Jiménez, Sinesio. «Perú: una modernización frustrada (1930-1991). Perú, reflexiones en el umbral de una nueva época. Instituto Democracia y Socialismo (IDS). Lima. Ciudadanos reales e imaginarios. Instituto Democracia y Socialismo (IDS). 1997. Lima.

26. Lynch, Nicolás. «La crisis de los partidos políticos». En: Grade. Lima. Mimeo.

27. Libro Blanco de la Defensa Nacional 2006

28. Mariátegui, José Carlos. Lo nacional y lo exótico. Editora Amauta, 1979, Lima, Perú.

29. Mariátegui, J.C. Peruanicemos al Perú, Editora Amauta, 1924, Lima, Perú.

30. Marx, Karl y F. Engels, Manifiesto del Partido Comunista, en K. Marx y F. Engels, Obras Escogidas, Tomo I, Editorial Progreso, Moscú.

31. Mazur, Gerhart. Simón Bolívar, University of New Mexico, Press. 1999.

32. Morote Solari, Fernando, Crl. EP, Manual para las Regiones Triunfadoras. Primera edición, 2003, Morote EDICIONES.

33. Mostajo Rossana, CEPAL, Autoridad social y la institucionalidad de la política social en el Perú.

34. Palacio Morena, Juan Ignacio. Europa en la globalización: Puntos fuertes y débiles. Universidad de Castilla-La Mancha. Papeles del Este Págs. 1-23. 2007. Madrid - España.

35. Pinillos, Gustavo. Identidad Nacional. 2005.

36. PNUD, P. d. La Identificación de las Potencialidades. Conceptos e Instrumentos. 2005, Lima - Perú.

37. PERU: Agenda y estrategia para el siglo 21, agenda Perú, noviembre 2000.

38. Promfret, Jhon. Reform hero. Gets life term for fraud, the Washington Post. 2000.

39. Ribeiro Darcy. El dilema de América Latina. Editorial Orbe, 1982.

40. Tanaka, Martín. El espejismo de la democracia. Instituto de Estudios peruanos. 1998. Lima.

41. Tejada Sergio. Los Estados Sordos: La Importancia de los Derechos Lingüísticos

42. Universidad Alas Peruanas. Como podemos ser un país más competitivo en el mundo actual. Logos del Contador. Año I - 2005.

43. Wash Catherine. La Interculturalidad en la Educación, Ministerio de Educación. 2001.

44. Wonacott, Peter. Shopping for china: A Scourge of the Rust Belt Offert Some Hope There Too. 2004.

45. Venero Gibaja, R. (31 de Julio de 1997). Producción, Productividad y Calidad de la Empresa. El Peruano.

46. Zhijun, Ling; Lian Ziang Feng Yun. 2005.

47. http://www.cies.org.pe/analisis/64/educacionpublica-regiones-mary-tam 